\section{Association of Male Gender and Younger Age at Diagnosis of Type 2 Diabetes with more Atherogenic Lipid Profile}

Weerarathna TP, Liyanage PLGC, Herath HMM

Faculty of Medicine, University of Ruhuna, PO Box 70, Galle, Sri Lanka

Contact information:

Thilak Priyantha Weerarathna

झ thilak.priyantha@yahoo.com

Background: Recognition of cardiovascular (CVD) risk profile and its patterns at the time of diagnosis of diabetes would enable adoption of most appropriate primary prevention strategies to reduce morbidity and mortality from vascular disease. We aimed to study the prevalence and pattern of modifiable CVD risk factors in patients at the time of diagnosis of type 2 diabetes.

Methods: In this single center, cross sectional study, 412 newly diagnosed patients with type 2 diabetes were studied on prevalence of low density lipoproteins (LDL), triglycerides (TG), high density lipoproteins (HDL) and systolic and diastolic blood pressure (SBP and $\mathrm{DBP})$. Prevalence of each risk factor was determined according to the American diabetes association criteria. Associations of each risk factor (dependent variable) with gender, age at onset of diabetes, waist circumference (WC) and body mass index (BMI) (independent variables) were studied using logistic regression.

Results: Males accounted for $71 \%$ of sample and Mean (SD) age 49(11) years. The most prevalent lipid abnormality was LDL > $100 \mathrm{mg}$ (81\%) and low HDL and raised TG were detected in 17.1\%, 18.5\%. $\mathrm{SBP}>140 \mathrm{~mm} \mathrm{Hg}$ and DBP > $90 \mathrm{~mm} \mathrm{Hg}$ were prevalent in $15 \%$ and $12 \%$. Male gender (odds ratio 7.98; 95\% confidence interval 4.39 to14.49) and younger age at onset of diabetes (odds ratio 0.95; $95 \%$ confidence interval 0.93 to 0.98 ) were significantly associated with lower HDL. The younger age was significantly associated with adverse

\section{Keywords}

type 2 diabetes, gender, CVD risk factors, atherogenic lipids, young onset diabetes 
TG levels (odds ratio $0.95 ; 95 \%$ confidence interval 0.93 to 0.95 ). Significant associations were detected with SBP and DBP with age (odds ratio $1.09 ; 95 \%$ confidence interval 1.05 to 1.13 ) and odds ratio 1.062 ; $95 \%$ confidence interval 1.006 to 1.121 ) respectively.

Discussion: Adverse LDL levels are present in 8 out of 10 patients at the time of diagnosis of diabetes. Modifiable CVD risk factors vary according to the age at onset of diabetes with more atherogenic dyslipidemia prevalent in younger and elevated blood pressure in older patients.

\section{Introduction}

Cardio-vascular diseases (CVD) contribute substantially to morbidity and mortality among individuals with type 2 diabetes (T2DM)(1). Dyslipidemia and hypertension are the major modifiable risk factors for this increased predilection (2). Lifestyle modifications and pharmacological interventions targeted at reducing blood pressure and altering adverse lipid profile have shown to reduce cardiovascular morbidity and mortality among individuals with $\operatorname{T2DM}(3,4)$.

Type 2 diabetes affects individuals in all age groups; the young, middle and elderly and also those with both lean and obese body habitus. Cardiovascular risk profile of an individual with diabetes is known to vary according to several factors including ethnicity and gender $(5,6)$. Studies conducted in multi ethnic settings revealed that individuals of South Asian ethnicity possess more adverse CVD risk factor profile and they succumb to cardiovascular diseases prematurely as compared to Caucasians(7). With rising burden of T2DM in South Asia, knowledge on the prevalence, pattern and associations of modifiable CVD risk factors in patients with newly diagnosed diabetes would enable clinicians to pay more attention on those with higher CVD risk. Implementation of targeted primary prevention strategies and management protocols could potentially delay or prevent adverse cardiovascular outcomes in patients with T2DM.

There is paucity of data on the prevalence of major modifiable risk factors and their variations according to gender, age at onset and type of obesity in patients with newly diagnosed T2DM in Sri Lanka. We aimed to study the prevalence and pattern of modifiable CVD risk factors including major lipid fractions and systolic and diastolic blood pressure in patients with newly diagnosed T2DM and to detect possible association of these risk factors with gender, age at onset of diabetes, central obesity (measured as waist circumference) and global obesity (measured as body mass index).

\section{Methodology}

This was a cross sectional study. We analyzed a database which included demographic, clinical and biochemical data of patients who underwent 
screening in a diabetes clinic at the time of first diagnosis of type 2 diabetes before commencing any hypoglycemic, anti -hypertensive or lipid lowering therapy. Ethical approval for the study was obtained from the local Ethics review committee. Data on their demographic (gender, age) clinical (height, weight, waist circumference, systolic and diastolic blood pressure) and biochemical (total cholesterol, low density lipoproteins, triglycerides, high density lipoprotein levels) parameters were analyzed. Optimal cutoff levels for blood pressure and lipids recommended by the American diabetes association (ADA) were used to determine the prevalence of each cardiovascular risk factor needing therapeutic intervention(8). The proportions with systolic blood pressure ( SBP> $140 \mathrm{~mm} \mathrm{Hg}$ ), diastolic blood pressure ( $D B P>90 \mathrm{~mm} \mathrm{Hg})$, low density lipoprotein level ( $\mathrm{LDL}>100 \mathrm{mg} / \mathrm{dL}$ ), triglycerides( TG> 150 $\mathrm{mg} / \mathrm{dL}$ ) and high density lipoprotein level ( $\mathrm{HDL}<$ $40 \mathrm{mg} / \mathrm{dL}$ in males and $<50 \mathrm{mg} / \mathrm{dL}$ in females) were estimated in the whole sample.

\section{Statistical analysis}

All numerical data were represented as mean (SD) and categorical data as percentages. Comparison of percentages with each individual CVD risk factors above the recommended level was carried out with chi $^{2}$ test. Logistic regression analysis was used to study the association of age, gender, BMI and waist circumference with each of the modifiable cardiovascular risk factor. Age adjusted odds ratios were calculated for each risk factor and level below 0.05 was taken as statistically significant.

\section{Results}

There were 412 patients with a majority (71\%) of males. The mean age (SD) and BMI (SD) were 44 (11) years and $24.5(3.3) \mathrm{kg} / \mathrm{m}^{2}$ respectively. The most frequently detected modifiable CVD risk factor was elevated LDL cholesterol level over 100 mg /
Table 1. Descriptive data of the patients with diabetes $(n=412)$.

\begin{tabular}{l|c|}
\multicolumn{1}{c|}{ Factor } & Percentage \\
\hline Age(years)* & $49(11)$ \\
\hline Gender (male) & $71.3 \%$ \\
\hline BMI (kg/m2)* & $24.5(3.3)$ \\
WC $(\mathrm{cm})^{*}$ & $91.1(8.9)$ \\
\hline SBP $(\mathrm{mmHg}))$ & $122(15)$ \\
\hline DBP $(\mathrm{mmHg}))$ & $77(9)$ \\
\hline TC $(\mathrm{mg} / \mathrm{dL}) *$ & $208.1(35.5)$ \\
\hline HDL $(\mathrm{mg} / \mathrm{dL}){ }^{*}$ & $49.4(7.7)$ \\
\hline LDL (mg/dL)* & $133.3(34.1)$ \\
\hline TG (mg/dL)* & $120.8(53.7)$ \\
\hline Proportion of suboptimal HDL & $17.1 \%$ \\
\hline Proportion of suboptimal LDL & $81.2 \%$ \\
\hline Proportion of suboptimal TG & $18.5 \%$ \\
\hline
\end{tabular}

* given mean (SD)

dL which was found in $81 \%$ of participants. Suboptimal levels of TG and HDL was found in $18.5 \%$ and $17.1 \%$ of patients. Descriptive data of the study participants are shown in table $\mathbf{1}$.

To study gender variations of modifiable cardiovascular risk factors, we compared the mean values of each risk factor in males and females included in the sample (table 2).

Females had higher age adjusted mean SBP and DBP and males had lower mean HDL levels. Mean levels of total cholesterol, LDL and triglycerides did not show any significant difference.

We carried out logistic regression analysis to study the association of each modifiable CVD risk factor (LDL > $100 \mathrm{mg} / \mathrm{dL}, \mathrm{TG}>150 \mathrm{mg} / \mathrm{dL}$ and $\mathrm{HDL}<$ $50 \mathrm{mg} / \mathrm{dL}$ in females and $<40 \mathrm{mg} / \mathrm{dL}$ in males and $S B P \geq 140 \mathrm{~mm} \mathrm{Hg}$ and $\mathrm{DBP} \geq 90 \mathrm{~mm} \mathrm{Hg}$ ) as the dependent variables and four clinical variables (gender, age at diagnosis, waist circumference (WC) and body mass index (BMI)) as independent variables.

Results of analysis of each CVD risk factors are 
shown in tables $\mathbf{3}$ - $\mathbf{6}$.

Males had higher odds of having LDL above 100 $\mathrm{mg} / \mathrm{dL}$ but it was not statistically significant. None of the other three clinical parameters studied showed a statistically significant association with elevated LDL cholesterol level over 100 mg / dL.

Of the four clinical variables, younger age and male gender had significantly higher odds ratios for low HDL. Although higher BMI was found to have a significant association with lower HDL levels, lower WC also showed a significant association with lower HDL cholesterol in this cohort.

Serum TG level above 150 mg / dL was significantly associated with younger age. None of the other three clinical variables had significant association with triglyceride level above $150 \mathrm{mg} / \mathrm{dL}$.

Table 2. Gender variations of mean values of each risk factor adjusted for age and BMI

\begin{tabular}{|c|c|c|c|}
\hline Factor & $\begin{array}{c}\text { Male } \\
\text { mean (SD) }\end{array}$ & $\begin{array}{c}\text { Female } \\
\text { mean (SD) }\end{array}$ & P value* \\
\hline SBP $(\mathrm{mmHg})$ & $120(14)$ & $124(17)$ & 0.03 \\
\hline $\mathrm{DBP}(\mathrm{mmHg})$ & $77(8)$ & $79(9)$ & 0.02 \\
\hline $\mathrm{TC}(\mathrm{mg} / \mathrm{dL})$ & $207.5(37)$ & $209.4(31.6)$ & 0.63 \\
\hline HDL $(\mathrm{mg} / \mathrm{dL})$ & $48.6(7.6)$ & $51.2(7.9)$ & $<0.01$ \\
\hline LDL $(\mathrm{mg} / \mathrm{dL})$ & $131.5(37)$ & $134.5(32.8)$ & 0.43 \\
\hline TG $(\mathrm{mg} / \mathrm{dL})$ & $123.2(55.4)$ & $115.0(49.0)$ & 0.16 \\
\hline
\end{tabular}

*student $\mathrm{t}$ test

Table 3. Association between clinical variables and high LDL

\begin{tabular}{|l|c|c|}
\hline Clinical risk factor & Odds ratio $(95 \% \mathbf{C I})$ & P value \\
\hline Age at diagnosis & $1.003(0.98$ to 1.03$)$ & 0.84 \\
\hline Gender (male) & $2.46(1.19$ to 5.07$)$ & 0.15 \\
\hline WC & $0.98(0.93$ to 1.03$)$ & 0.4 \\
\hline BMI & $0.99(0.87$ to 1.12$)$ & 0.86 \\
\hline
\end{tabular}

Table 4. Association between clinical variables and low HDL

\begin{tabular}{|l|c|c|}
\hline \multicolumn{1}{|c|}{ Clinical risk factor } & Odds ratio $(95 \% \mathbf{C l})$ & P value \\
\hline Age at diagnosis & $0.95(0.93$ to 0.98$)$ & $<0.01$ \\
\hline Gender (male) & $7.98(4.39$ to 14.49$)$ & $<0.01$ \\
\hline WC & $0.95(0.91$ to 0.99$)$ & 0.03 \\
\hline BMI & $1.139(1.077$ to 1.288$)$ & 0.04 \\
\hline
\end{tabular}

Table 5. Association between clinical variables and high TG

\begin{tabular}{|l|c|c|}
\hline Clinical risk factor & Odds ratio $(95 \% \mathrm{Cl})$ & P value \\
\hline Age at diagnosis & $0.95(0.93$ to 0.98$)$ & $<0.01$ \\
\hline Gender (male) & $0.73(0.39$ to 1.36$)$ & 0.32 \\
\hline WC & $1.02(0.98$ to 1.07$)$ & 0.36 \\
\hline BMI & $1.003(0.888$ to 1.133$)$ & 0.96 \\
\hline
\end{tabular}

Table 6. Association between clinical variables and elevated SBP

\begin{tabular}{|l|c|c|}
\hline Clinical risk factor & Odds ratio $(95 \% \mathbf{C l})$ & P value \\
\hline Age at diagnosis & $1.09(1.05$ to 1.13$)$ & $<0.01$ \\
\hline Gender (male) & $1.16(0.53$ to 2.55$)$ & 0.71 \\
\hline WC & $0.98(0.92$ to 1.04$)$ & 0.53 \\
\hline BMI & $1.02(0.86$ to 1.20$)$ & 0.82 \\
\hline
\end{tabular}


Table 7. Association between clinical variables and elevated DBP

\begin{tabular}{|l|c|c|}
\hline \multicolumn{1}{|c|}{ Clinical risk factor } & Odds ratio $(\mathbf{9 5} \% \mathbf{C l})$ & P value \\
\hline Age at diagnosis & $1.062(1.006$ to 1.121$)$ & 0.03 \\
\hline Gender (male) & $1.28(0.39$ to 4.11$)$ & 0.68 \\
\hline WC & $1.01(0.92$ to 1.11$)$ & 0.86 \\
\hline BMI & $0.98(0.76$ to 1.25$)$ & 0.86 \\
\hline
\end{tabular}

Higher age at diagnosis was found to be significantly associated with SBP over $140 \mathrm{~mm} \mathrm{Hg}$. Although male gender and higher BMI showed higher odds of having SBP over $140 \mathrm{~mm} \mathrm{Hg}$ none of them were statistically significant.

Out of the four parameters only the age at diagnosis showed statistically significant association with diastolic blood pressure over $90 \mathrm{~mm} \mathrm{Hg}$.

\section{Discussion}

Findings of this study reveal that there is a high prevalence (81. $2 \%$ ) of adverse level of LDL cholesterol (over $100 \mathrm{mg} / \mathrm{dL}$ ) at the time of diagnosis of diabetes. Although more males had LDL above 100 $\mathrm{mg} / \mathrm{dL}$ than females, no significant gender variation was observed. But male gender was associated with significantly higher odds of having lower HDL cholesterol level. Mean levels of SBP and DBP were significantly higher in females and mean HDL levels were significantly lower in males. Males with newly diagnosed T2DM compared to females have non-significantly higher odds of possessing all major modifiable CVD risk factors except higher TG level. Of the clinical parameters, younger age at onset of diabetes revealed a significant association with higher TG and lower HDL cholesterol levels. BMI revealed a positive association with SBP, TG and low HDL but a statistically significant association of BMI was only seen with lower HDL cholesterol level.
Gender variations of cardiovascular risk factors among patients with diabetes reported in previous studies reveal a comparatively adverse risk profile amongst females(9). This has been postulated as a possible cause for higher cardiovascular morbidity and mortality in females with diabetes. Our study included a majority (71\%) of males and we found higher mean levels of SBP and DBP levels in females and lower HDL in males. Relatively lower proportion of females (29\%) included in this study sample could have affected these results.

According to studies conducted in multi ethnic settings, there are significant variations in the prevalence and pattern of CVD risk factors among individuals with diabetes (10). A meta-analysis of lipid parameters of 24, 000 subjects has reported that Asian Indians with and without diabetes compared to Caucasians and Chinese have significantly higher odds of lower HDL cholesterol and higher TG levels(11). They also reported significantly lower HDL cholesterol in males compared to females of Asian Indian ethnicity. A study conducted in multiethnic population in the United Kingdom have revealed comparatively worse lipid profiles with lower HDL cholesterol levels and higher CVD event rate among the South Asians compared to Caucasians(7). A study conducted in Singapore among patients with type 2 diabetes from different Asian ethnicities have reported that Asian Indians have significantly lower HDL cholesterol levels compared to Malays and Chinese patients(12). One previous study of 592 patients with newly diagnosed T2DM conducted in Sri Lanka has reported similar prevalence of high TG (14\%) and low HDL (12\%) but they do not report on the prevalence of high LDL among the study participants (13).

Pattern of dyslipidemia especially the high prevalence of adverse LDL cholesterol levels in more than $80 \%$ of individuals with diabetes at the time of diabetes in this study has several management implications. It is well established that adverse levels of LDL cholesterol is strongly associated with all types 
of CVD including coronary artery disease (CAD) , cerebrovascular disease (CVD) and peripheral artery disease (PAD). American diabetes association advocates that the optimal level of LDL cholesterol to prevent CVD in patients with diabetes should be less than $100 \mathrm{mg} / \mathrm{dL}$. Therefore, based on the single risk factor modification approach, findings of this study would compel clinicians to start statin therapy for $80 \%$ of patients at the time of diagnosis of T2DM. However, with the introduction of total cardiovascular risk management approach, there is a tendency to avoid commencement of pharmacotherapy based on a single risk factor. Total CVD risk management approach recommends initiation of pharmacotherapy such as statins or aspirin based on the category (low, moderate or high) of cardiovascular risk of the individual. The latter is calculated using an appropriate chart or a score such as Framingham risk chart or United Kingdom prospective diabetes study (UKPDS) risk engine respectively.

Most studies on the epidemiology of lipids among individuals with newly diagnosed diabetes are conducted in the Caucasians and they have shown that LDL cholesterol levels in the diabetic sub population are not significantly different from the non-diabetic subjects. Mean LDL cholesterol levels in the newly diagnosed males and females with T2DM recruited for the UKPDS was 139 and $153 \mathrm{mg} / \mathrm{dL}(\mathrm{p}=<0.05) \mathrm{re}$ spectively(14). Newly diagnosed patients with T2DM in this study revealed similar mean LDL cholesterol level (133 mg/ dL), but unlike in UKPDS study which showed significantly higher mean LDL cholesterol in females, more male patients had adverse LDL cholesterol in this study. Observation of more than $80 \%$ having higher levels of LDL in this study could be due to the lower cut off limit used to define the undesirable LDL cholesterol according to the recent guidelines. Lower prevalence rates of adverse LDL cholesterol levels in some previous studies could be due to the higher cut-off levels used to categorize adverse level of LDL cholesterol according to older guidelines.
This study also revealed a statistically significant association of younger age at onset of diabetes with higher TG levels (OR - 0.95, Cl 0.93 to 0.98, p $<0.01$ ) and lower HDL (OR - 0.95, Cl 0.93 to 0.98, $\mathrm{p}<0.01)$. High TG level and low HDL cholesterol level is a surrogate marker of insulin resistance and is also considered as a hall mark of atherogenic dyslipidemia (15). Studies using TG/ HDL ratio as the Atherogenic plasma index (API) have shown that it correlate positively with carotid intima media thickness (CIMT), a surrogate marker of atherosclerosis (16).

Finding of atheogenic dyslipidemia among the younger age at onset of T2DM has several implications. Although statin therapy reduces elevated LDL cholesterol with clinically significant reductions in all forms of CVD, to date, attempts to find a safe and effective pharmacological agent to raise low HDL levels which lead to favorable clinical outcomes have not been very successful (17). In the absence of a safe and effective pharmacotherapy to optimize atherogenic dyslipidemia, clinical guidelines emphasize the importance of non-pharmacological interventions to achieve favorable HDL cholesterol (15). Findings of this study emphasize the need for implementation of intensive non-pharmacological approaches aimed at optimizing the atherogenic lipids for younger patients with diabetes from the time of diagnosis of diabetes.

High blood pressure, defined as systolic and blood pressures over 140 and $90 \mathrm{~mm} \mathrm{Hg}$, were found to be less prevalent (12-15\%) than the adverse lipids (80\%) among subjects with diabetes in this study. The prevalence of hypertension in this study is lower than the reported prevalence of hypertension in the population. A nationwide study in a sample of 5000 people conducted in 2005 had revealed 23\% prevalence of hypertension (18). The lower prevalence of hypertension among newly diagnosed patients with diabetes could be due to the relatively more number of younger patients with diabetes included in our sample and the smaller sample size. As with 
adverse lipids, male gender was associated with non-significant, higher odds of having both systolic and diastolic blood pressure in this study. Of the clinical parameters, age at onset of diabetes showed significantly higher odds of having higher SBP and DBP implying that older age at onset of T2DM is more likely to be associated with higher systolic and diastolic blood pressure values.

Major strength of this study is the inclusion of patients at different ages of diabetes without commencing any non-pharmacological or pharmacological therapy to control lipids, blood pressure or blood sugar making them truly newly diagnosed without any lifestyle of pharmacological intervention. As our findings are derived from a single center, generalization of these findings for the population needs population based or representative multi center studies. Inclusion of a majority (71\%) of males is a one limitation of our study. Although mean SBP and DBP levels were higher among females, we could not find gender as a significant determinant of any CVD risk factor except for lower HDL in males on logistic regression. Smaller proportion of females (29\%) included could have affected the findings of this study.

\section{Conclusion}

In summary, findings of this study highlight three major patterns of CVD risk factors prevalent among patients at the time of diagnosis of T2DM. Of them, the therapeutically most relevant finding is the need to initiate statin therapy in eight out of ten patients at the time of diagnosis for primary prevention of CVD. The other two observations include the statistically significant associations of atherogenic dyslipidemia with lower HDL and higher TG levels with younger age at onset of diabetes and male gender. Finding of both SBP and DBP having a statistically significant association with increasing age at the time of diagnosis of diabetes is expected as both forms of blood pressure are known to rise with advancing age.

\section{References}

1. Candido R, Srivastava P, Cooper ME, Burrell LM. Diabetes mellitus: a cardiovascular disease. Current opinion in investigational drugs (London, England : 2000). 2003;4(9):1088-1094.

2. Baldwin MD. Assessing cardiovascular risk factors and selecting agents to successfully treat patients with type 2 diabetes mellitus. The Journal of the American Osteopathic Association. 2011;111(7 Suppl 5):S2-12.

3. Toth PP, Simko RJ, Palli SR, Koselleck D, Quimbo RA, Cziraky MJ. The impact of serum lipids on risk for microangiopathy in patients with type 2 diabetes mellitus. Cardiovascular diabetology. 2012;11:109.

4. Long GH, Cooper AJ, Wareham NJ, Griffin SJ, Simmons RK. Healthy behavior change and cardiovascular outcomes in newly diagnosed type 2 diabetic patients: a cohort analysis of the ADDITION-Cambridge study. Diabetes Care. 2014;37(6):17121720.

5. Wang Y, Katzmarzyk PT, Horswell R, Zhao W, Li W, Johnson J, Ryan $\mathrm{DH}, \mathrm{Hu}$ G. Racial disparities in cardiovascular risk factor control in an underinsured population with Type 2 diabetes. Diabet Med. 2014;31(10):1230-1236.

6. Haidinger T, Zweimuller M, Stutz L, Demir D, Kaider A, StrametzJuranek J. Effect of gender on awareness of cardiovascular risk factors, preventive action taken, and barriers to cardiovascular health in a group of Austrian subjects. Gend Med. 2012;9(2):94-102.

7. Bellary S, O'Hare JP, Raymond NT, Mughal S, Hanif WM, Jones A, Kumar S, Barnett AH. Premature cardiovascular events and mortality in south Asians with type 2 diabetes in the United Kingdom Asian Diabetes Study - effect of ethnicity on risk. Curr Med Res Opin. 2010;26(8):1873-1879.

8. Executive summary: Standards of medical care in diabetes--2014. Diabetes Care. 2014;37 Suppl 1:S5-13.

9. Kautzky-Willer A, Kamyar MR, Gerhat D, Handisurya A, Stemer G, Hudson S, Luger A, Lemmens-Gruber R. Sex-specific differences in metabolic control, cardiovascular risk, and interventions in patients with type 2 diabetes mellitus. Gend Med. 2010;7(6):571-583.

10. Davis TM, Cull CA, Holman RR. Relationship between ethnicity and glycemic control, lipid profiles, and blood pressure during the first 9 years of type 2 diabetes: U.K. Prospective Diabetes Study (UKPDS 55). Diabetes Care. 2001;24(7):1167-1174.

11. Zhang L, Qiao Q, Tuomilehto J, Janus ED, Lam TH, Ramachandran A, Mohan V, Stehouwer CD, Dong Y, Nakagami T, Onat A, Soderberg S. Distinct ethnic differences in lipid profiles across glucose categories. J Clin Endocrinol Metab. 2010;95(4):17931801.

12. Hughes K, Yeo PP, Lun KC, Thai AC, Sothy SP, Wang KW, Cheah JS, Phoon WO, Lim P. Cardiovascular diseases in Chinese, Malays, and Indians in Singapore. II. Differences in risk factor levels. Journal of epidemiology and community health. 1990;44(1):29-35. 
13. Weerasuriya N, Siribaddana S, Dissanayake A, Subasinghe Z, Wariyapola D, Fernando DJ. Long-term complications in newly diagnosed Sri Lankan patients with type 2 diabetes mellitus. QJM : monthly journal of the Association of Physicians. 1998;91(6):439-443.

14. U.K. Prospective Diabetes Study 27. Plasma lipids and lipoproteins at diagnosis of NIDDM by age and sex. Diabetes Care. 1997;20(11):1683-1687.

15. Eeg-Olofsson K, Gudbjornsdottir S, Eliasson B, Zethelius B, Cederholm J, on behalf of the NDR. The triglycerides-to-HDLcholesterol ratio and cardiovascular disease risk in obese patients with type 2 diabetes: An observational study from the Swedish National Diabetes Register (NDR). Diabetes research and clinical practice. 2014.

16. Li X, Deng YP, Yang M, Wu YW, Sun SX, Sun JZ. Triglyceride to high-density lipoprotein cholesterol ratio and carotid intimamedial thickness in Chinese adolescents with newly diagnosed type 2 diabetes mellitus. Pediatr Diabetes. 2015

17.Zhu XW, Deng FY, Lei SF. Meta-analysis of Atherogenic Index of Plasma and other lipid parameters in relation to risk of type 2 diabetes mellitus. Primary care diabetes. 2014.

18. Katulanda P, Ranasinghe $P$, Jayawardena R, Constantine GR, Rezvi Sheriff MH, Matthews DR. The prevalence, predictors and associations of hypertension in Sri Lanka: a cross-sectional population based national survey. Clinical and experimental hypertension. 2014;36(7):484-491.

\section{Comment on this article:}

\section{Qf $[$ in $8+\mathbf{S} P$}

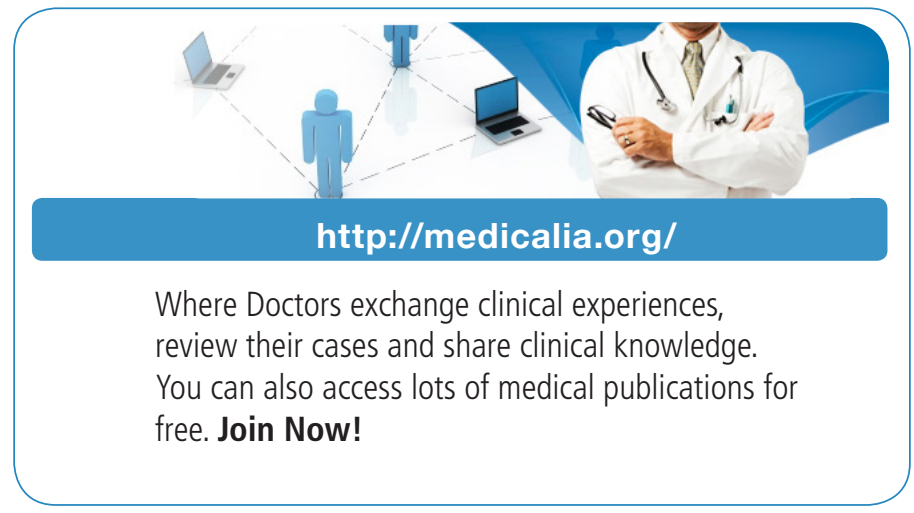

\section{Publish with iMedPub}

\section{http://www.imed.pub}

International Archives of Medicine is an open access journal publishing articles encompassing all aspects of medical science and clinical practice. IAM is considered a megajournal with independent sections on all areas of medicine. IAM is a really international journal with authors and board members from all around the world. The journal is widely indexed and classified Q1 in category Medicine. 